\title{
AN EXTREMAL METHOD ON ARBITRARY RIEMANN SURFACES
}

\author{
BY \\ LEO SARIO
}

Introduction. A general problem in the theory of abstract Riemann surfaces is to construct a function which minimizes the value of a given functional $m$ defined on a class $F$ of functions on a given Riemann surface $R$. The minimizing function is, at each point of the surface, a new functional of $F$ and $m$. We shall give, by two typical examples, a method for constructing such functionals.

For $F$, we shall first take the class $\{P\}$ of analytic functions with the expansion

$$
P=p+i \bar{p}=\frac{1}{z^{k}}+\sum_{1}^{\infty} a_{\nu} z^{\nu}
$$

in a fixed parameter disc $|z| \leqq 1$ and with a single-valued real part $p$ on $R$. For the quantity $m$ to be minimized, we take

$$
m_{\lambda}(p)=2 \pi \lambda k \alpha_{k}+\int_{\beta} p d \bar{s}
$$

Here $\lambda$ is a real parameter belonging to the interval $(-1,1), \alpha_{k}$ stands for $\operatorname{Re}\left(a_{k}\right)$, and $\beta$ is the (ideal) boundary of $R$. The integral along $\beta$ is defined as the limit of integrals along boundaries of exhausting domains. We shall prove that there exists in $\{P\}$ a unique function $P_{\lambda}$, called the principal function,

$$
P_{\lambda}=p_{\lambda}+i \bar{p}_{\lambda}=\frac{1}{z^{k}}+\sum_{1}^{\infty} a_{\lambda \nu} z^{\nu}
$$

which minimizes $m_{\lambda}$ for a fixed $k$. This function is a linear combination of $P_{-1}$ and $P_{1}$. The minimum of $m_{\lambda}$ can be expressed solely in terms of $\alpha_{-1 k}$ and $\alpha_{1 k}$. For any $p$, the deviation of $m_{\lambda}$ from this minimum is equal to the Dirichlet integral of $p-p_{\lambda}$. The coefficient $\alpha_{k}$ is minimized by $\alpha_{1 k}$ and maximized by $\alpha_{-1 k}$ for functions with $\int_{\beta} p d \overline{0} \leqq$. The $P$-span

$$
\sigma_{P}=\alpha_{-1 k}-\alpha_{1 k}
$$

is equal, up to a constant factor, to the minimum of $\int_{\beta} p d p$. This minimum is attained for $p_{0}=\left(p_{1}+p_{-1}\right) / 2$. All functions $p$ with $\int_{\beta} p d \bar{p} \leqq 0$ coincide if and only if the $P$-span vanishes. The function $p_{-1}-p_{1}$ is bounded and minimizes

Presented to the Society, May 3, 1952; received by the editors February 18, 1952. 
the Dirichlet integral among (normalized) single-valued harmonic functions on $R$. The minimum is, up to a constant factor, the reciprocal of the $P$-span.

For a second class, we shall consider the subclass $\{Q\}$ of $\{P\}$ defined by the additional property that the period of the imaginary part $\bar{q}$ vanishes along every cycle dividing $R$ into disjoint parts. In this class, there is again a unique principal function $Q_{\lambda}$ which minimizes $m_{\lambda}(q)$. The properties attributed above to $P_{\lambda}$ are shared by $Q_{\lambda}$ when $\alpha_{\lambda k}$ and the $Q$-span $\sigma_{Q}=\alpha_{-1 k}-\alpha_{1 k}$ are taken with respect to $\{Q\}$. On planar surfaces, the functions $Q$ are single-valued, and $Q_{1}$ and $Q_{-1}$ (for $k=1$ ) map $R$ onto the vertical and horizontal slit domains. The $Q$-span coincides with the span introduced by Schiffer for multiplyconnected plane domains [14].

The problems of maximizing $\alpha$, of minimizing $\int q d \bar{q}$, and of finding the connection between these extrema were introduced by de Possel [6], Grunsky [2], and Schiffer [14] respectively. These authors gave the solution for plane domains. The contributions of the present paper to these problems are: (1) By the choice of $m_{\lambda}$ as a combination of $\alpha_{k}$ and $\int q d \bar{q}$, the three problems are unified and solved simultaneously. (2) The solution is given also for the more general class $\{P\}$, that is, without assumptions on the imaginary part. (3) The problems are formulated and solved for arbitrary Riemann surfaces.

These results have applications to the classification theory of Riemann surfaces. With notations introduced in $\$ 4$, the following relations will be proved:

$$
\begin{gathered}
O_{H B} \subset O_{H B D}=O_{H D}=S_{P}, \\
O_{K B} \subset O_{K B D}=O_{K D}=S_{Q}, \\
O_{W E}=O_{A B} \subset O_{A D},
\end{gathered}
$$

and, for planar surfaces,

$$
O_{A B} \subset O_{A B D}=O_{A D}=O_{S E}=S_{Q} .
$$

These relations include those established by Ahlfors-Beurling [1], Lehto [3], Lokki [4], Parreau [5], Royden [7], and Virtanen [15], using primarily the method of extremal length and Dirichlet's principle.

The extremal method suggested in the present paper can also be used in the investigation of bounded schlicht functions and functions with a singlevalued modulus. It leads, in a natural way, to relations concerning the capacity of a boundary component of an arbitrary Riemann surface [13]. Furthermore, it yields a general operator $L_{\lambda}$ [12], applicable in the linear operator method [10]; there the quantity $m$ to be minimized by the principal function characterizes its boundary behavior. A survey of the above methods with applications is presented in [11].

1. The class $\{P\}$. Let $R$ be an arbitrary open Riemann surface. Consider the class $\{P\}$ of analytic functions with the expansion 


$$
P=p+i \bar{p}=\frac{1}{z^{k}}+\sum_{1}^{\infty} a_{\nu} z^{\nu}
$$

in a fixed parameter disc $D:|z| \leqq 1$ and with a single-valued real part $p$ on $R$. Here $k$ is a fixed positive integer. The functions $P$ are supposed to be free from singularities other than $z=0$. Write

$$
P_{\lambda}=p_{\lambda}+i \bar{p}_{\lambda}=\frac{1}{z^{k}}+\sum_{1}^{\infty} a_{\lambda \nu} z^{\nu}
$$

and set $\alpha_{\lambda k}=\operatorname{Re}\left(a_{\lambda k}\right)$.

THEOREM 1. In the class $\{P\}$, there is a uniquely determined principal function $P_{\lambda}$ which minimizes

$$
m_{\lambda}(p)=\lambda \cdot 2 \pi k \alpha_{k}+\int_{\beta} p d \bar{p} .
$$

This minimizing function has the following properties:

$1^{\circ}$. The deviation of $m_{\lambda}$ from the minimum is given by the Dirichlet integral, that is,

$$
m_{\lambda}(p)=m_{\lambda}\left(p_{\lambda}\right)+D\left(p-p_{\lambda}\right) .
$$

$2^{\circ}$. The minimum for any $\lambda$ in the interval $(-1,1)$ can be written in terms of $\alpha_{1 k}$ and $\alpha_{-1 k}$ and has the value

$$
m_{\lambda}\left(p_{\lambda}\right)=2^{-1} \pi k\left[(1+\lambda)^{2} \alpha_{1 k}-(1-\lambda)^{2} \alpha_{-1 k}\right] .
$$

$3^{\circ}$. The function $P_{\lambda}$ is a linear combination of $P_{1}$ and $P_{-1}$ as follows:

$$
P_{\lambda}=\frac{1+\lambda}{2} P_{1}+\frac{1-\lambda}{2} P_{-1} \text {. }
$$

Proof. Suppose first that $R$ is bounded by a finite set $\beta$ of closed analytic Jordan curves. In the class $\{p\}$, consisting of the real parts of functions $\{P\}$, there are two particular functions $p_{1}$ and $p_{-1}$ determined by the conditions

$$
\begin{aligned}
p_{1} & =\text { const. on } \beta, \\
\frac{\partial p_{-1}}{\partial n} & =0 \text { on } \beta,
\end{aligned}
$$

where $\partial / \partial n$ is the normal derivative. These functions can be constructed by well known methods (for example [10]). Let

$$
p_{\lambda}=\frac{1+\lambda}{2} p_{1}+\frac{1-\lambda}{2} p_{-1} .
$$


For any $p$ on $R$, we have, writing $p-p_{\lambda}=h$,

$$
m_{\lambda}(p)=2 \pi \lambda k \alpha_{k}+\int_{\beta} p_{\lambda} d \bar{p}_{\lambda}+\int_{\beta} p_{\lambda} d \bar{h}+\int_{\beta} h d \bar{p}_{\lambda}+D(h) .
$$

Here

$$
\begin{aligned}
\int_{\beta} p_{\lambda} d \bar{\lambda}_{\lambda} & =\frac{1-\lambda^{2}}{4} \int_{\beta} p_{-1} d p_{1}+p_{1} d \bar{p}_{-1}=\frac{1-\lambda^{2}}{4} \int_{\beta} p_{-1} d p_{1}-p_{1} d p_{-1} \\
& =\frac{1-\lambda^{2}}{4} \int_{\alpha} p_{-1} d p_{1}-p_{1} d p_{-1}=\frac{1-\lambda^{2}}{4} \int_{\alpha} p_{-1} d p_{1}+p_{-1} d p_{1} \\
& =\frac{1-\lambda^{2}}{4} \operatorname{Im} \int_{\alpha} P_{-1} d P_{1},
\end{aligned}
$$

where the transfer from $\beta$ to $\alpha:|z|=1$ is justified by Green's formula. It follows, by (2), that

$$
\int_{\beta} p_{\lambda} d \bar{p}_{\lambda}=2^{-1} \pi k\left(1-\lambda^{2}\right)\left(\alpha_{1 k}-\alpha_{-1 k}\right)
$$

Furthermore,

$$
\int_{\beta} p_{\lambda} d \bar{h}=\frac{1-\lambda}{2} \int_{\beta} p_{-1} d \bar{h}=\frac{1-\lambda}{2} \operatorname{Im} \int_{\alpha} P_{-1} d H=\pi k(1-\lambda)\left(\alpha_{k}-\alpha_{\lambda k}\right),
$$

and similarly,

$$
\int_{\beta} h d \bar{p}_{\lambda}=\frac{1+\lambda}{2} \int_{\beta} h d \bar{p}_{1}=\frac{1+\lambda}{2} \operatorname{Im} \int_{\alpha} H d P_{1}=\pi k(1+\lambda)\left(\alpha_{\lambda k}-\alpha_{k}\right) .
$$

Adding these expressions, and making use of the formula

$$
\alpha_{\lambda k}=\frac{1+\lambda}{2} \alpha_{1 k}+\frac{1-\lambda}{2} \alpha_{-1 k},
$$

we have the important relation

$$
m_{\lambda}(p)=2^{-1} \pi k\left[(1+\lambda)^{2} \alpha_{1 k}-(1-\lambda)^{2} \alpha_{-1 k}\right]+D\left(p-p_{\lambda}\right) .
$$

The theorem then follows for the case of a region with analytic boundary.

For $\lambda=1,-1$ we find in particular

$$
2 \pi k \alpha_{k}+\int_{\beta} p d \overline{2} \geqq 2 \pi k \alpha_{1 k}, \quad-2 \pi k \alpha_{k}+\int_{\beta} p d \overline{2} \geqq-2 \pi k \alpha_{-1 k},
$$

and consequently

$$
\alpha_{1 k} \leqq \alpha_{k} \leqq \alpha_{-1 k}
$$




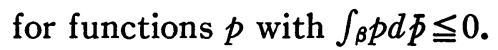

Now let $R$ be an arbitrary open Riemann surface of finite or infinite genus and let $\left\{R_{n}\right\}$ be an exhaustion of $R$, where $R_{n}$ is bounded by a finite set $\beta_{n}$ of closed analytic Jordan curves. Suppose that

$$
P_{\lambda n}=\frac{1}{z^{k}}+\sum_{\nu=1}^{\infty} a_{\lambda n \nu} z^{\nu}
$$

is the minimizing function for $R_{n}$. It follows, by (11), for $n \geqq N$ that

$$
D_{N}\left(p_{\lambda n}-p_{\lambda N}\right)=m_{\lambda N}\left(p_{\lambda n}\right)-m_{\lambda N}\left(p_{\lambda N}\right),
$$

where the Dirichlet integral $D_{N}$ is taken over $R_{N}$, and where $m_{\lambda N}$ is (3) for $R_{N}$. Since the Dirichlet integral of $p_{\lambda_{n}}$ over $R_{n}-R_{N}$ is non-negative, we have

$$
\int_{\beta_{N}} p_{\lambda n} d \bar{p}_{\lambda n} \leqq \int_{\beta_{n}} p_{\lambda n} d \bar{\lambda}_{\lambda n}
$$

and

$$
m_{\lambda N}\left(p_{\lambda_{n}}\right) \leqq m_{\lambda n}\left(p_{\lambda n}\right)
$$

Hence, by (11),

$$
D_{N}\left(p_{\lambda n}-p_{\lambda N}\right) \leqq 2^{-1} \pi k\left[(1+\lambda)^{2}\left(\alpha_{1 n k}-\alpha_{1 N k}\right)-(1-\lambda)^{2}\left(\alpha_{-1 n k}-\alpha_{-1 N k}\right)\right] .
$$

Now we make use of our assumption $|\lambda| \leqq 1$. It implies, by (13) and (10),

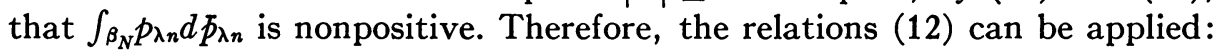

$$
\alpha_{1 n k} \leqq \alpha_{-1 N k}, \quad \alpha_{-1 n k} \geqq \alpha_{1 N k} .
$$

It follows that

$$
D_{N}\left(p_{\lambda n}-p_{\lambda N}\right) \leqq \pi k\left(1+\lambda^{2}\right)\left(\alpha_{-1 N k}-\alpha_{1 N k}\right) .
$$

Thus, the class $\left\{p_{\lambda_{n}}\right\}$ for $n \geqq N$ is a normal family in $R_{N}$. Since $R_{n} \rightarrow R$, a subsequence, say $\left\{p_{\lambda_{n}}\right\}$, tends to a function $p_{\lambda}$ in $R$,

$$
p_{\lambda}=\lim _{n \rightarrow \infty} p_{\lambda n} \text {. }
$$

By the minimum property of $p_{\lambda n}$ in $R_{n}$,

$$
m_{\lambda n}\left(p_{\lambda n}\right) \leqq m_{\lambda n}\left(p_{\lambda(n+1)}\right) \leqq m_{\lambda(n+1)}\left(p_{\lambda(n+1)}\right) .
$$

This yields

$$
\lim _{n \rightarrow \infty} m_{\lambda n}\left(p_{\lambda n}\right) \leqq \inf m_{\lambda}(p) \leqq m_{\lambda}\left(p_{\lambda}\right),
$$

where $\inf m_{\lambda}(p)$ is (3) for the class $\{p\}$ on $R$. In fact, every $p$ on $R$ is an admissible function on $R_{n}$, which implies that $m_{\lambda_{n}}\left(p_{\lambda_{n}}\right) \leqq m_{\lambda}(p)$. Thus the first inequality is established. On the other hand, 


$$
m_{\lambda}\left(p_{\lambda}\right)=\lim _{n \rightarrow \infty} m_{\lambda n}\left(p_{\lambda}\right)=\lim _{n \rightarrow \infty} \lim _{m \rightarrow \infty} m_{\lambda n}\left(p_{\lambda m}\right) \leqq \lim _{m \rightarrow \infty} m_{\lambda m}\left(p_{\lambda m}\right) .
$$

Therefore,

$$
\min m_{\lambda}(p)=m_{\lambda}\left(p_{\lambda}\right)=\lim _{n \rightarrow \infty} m_{\lambda n}\left(p_{\lambda_{n}}\right) .
$$

For any $p=p_{\lambda}+h$ and any real $\epsilon$,

$$
\begin{aligned}
m_{\lambda}\left(p_{\lambda}+\epsilon h\right)= & m_{\lambda}\left(p_{\lambda}\right) \\
& +\epsilon\left\{\epsilon D(h)+\left[\lambda \cdot 2 \pi k\left(\alpha_{k}-\alpha_{\lambda k}\right)+\int_{\beta}\left(p_{\lambda} d \bar{h}+h d p_{\lambda}\right)\right]\right\} .
\end{aligned}
$$

The minimum property of $p_{\lambda}$ implies that

$$
\lambda \cdot 2 \pi k\left(\alpha_{k}-\alpha_{\lambda k}\right)+\int_{\beta}\left(p_{\lambda} d \bar{h}+h d \bar{p}_{\lambda}\right)=0,
$$

for otherwise $m_{\lambda}\left(p_{\lambda}+\epsilon h\right)-m_{\lambda}\left(p_{\lambda}\right)$ would change its sign with $\epsilon$ for sufficiently small $|\epsilon|$. Setting $\epsilon=1$, we have equation (4):

$$
m_{\lambda}(p)=m_{\lambda}\left(p_{\lambda}\right)+D\left(p-p_{\lambda}\right) .
$$

The uniqueness of $p_{\lambda}$ now follows. Let $p_{\lambda}^{\prime}$ and $p_{\lambda}^{\prime \prime}$ be two minimizing functions. Then

$$
m_{\lambda}\left(p_{\lambda}^{\prime}\right)=m_{\lambda}\left(p_{\lambda}^{\prime \prime}\right)+D\left(p_{\lambda}^{\prime}-p_{\lambda}^{\prime \prime}\right)=m_{\lambda}\left(p_{\lambda}^{\prime \prime}\right)
$$

and

$$
D\left(p_{\lambda}^{\prime}-p_{\lambda}^{\prime \prime}\right)=0 .
$$

Thus, since $p_{\lambda}^{\prime}-p_{\lambda}^{\prime \prime}=0$ at $z=0$, we have $p_{\lambda}^{\prime} \equiv p_{\lambda}^{\prime \prime}$. In particular, the original sequence $p_{\lambda_{n}}$ converges.

By (11) applied to $R_{n}$ and by (15), equation (5) follows for $R$. Similarly, the equation (9), defining the approximating functions $p_{\lambda_{n}}$ on $R_{n}$, continues to hold for the limit function on $R$. The theorem is thus proved for an arbitrary $R$.

2. Other extremal properties of the principal function $p_{\lambda}$. In order to apply Theorem 1, we combine (4) and (5) to obtain the fundamental formula

$$
m_{\lambda}(p)=2^{-1} \pi k\left[(1+\lambda)^{2} \alpha_{1 k}-(1-\lambda)^{2} \alpha_{-1 k}\right]+D\left(p-p_{\lambda}\right) .
$$

We define the $P$-span $\sigma_{P}$ by

$$
\sigma_{P}=\alpha_{-1 k}-\alpha_{1 k} .
$$

Theorem 2. $P_{1}$ minimizes and $P_{-1}$ maximizes $\alpha_{k}$ among functions $P$ with

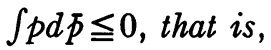




$$
\alpha_{1 k} \leqq \alpha_{k} \leqq \alpha_{-1 k}
$$

The boundary integral for $p_{\lambda}$ has the nonpositive value

$$
\int_{\beta} p_{\lambda} d \bar{p}_{\lambda}=2^{-1} \pi k\left(\lambda^{2}-1\right) \sigma_{P} \leqq 0 .
$$

In particular,

$$
\begin{gathered}
\int_{\beta} p_{1} d p_{1}=\int_{\beta} p_{-1} d p_{-1}=0 \\
\int_{\beta} p_{0} d \bar{p}_{0}=\min \left(\int_{\beta} p d \bar{p}\right)=-2^{-1} \pi k \sigma_{P} \leqq 0
\end{gathered}
$$

where $p_{0}=2^{-1}\left(p_{1}+p_{-1}\right)$.

The function $p_{-1}-p_{1}$ is bounded on $R$.

Proof. (18) follows from (17) for $\lambda=1,-1$. For $p=p_{\lambda},(17)$ gives (19) and (20). In $R_{n}-D$, we have $\left|p_{-1 n}\right| \leqq \max _{\alpha}\left|p_{-1 n}\right|$. In fact, if $p_{-1 n}$ is continued harmonically on the "double" surface formed by symmetrizing $R_{n}-D$ with respect to $\beta_{n}$, it assumes identical values on the two copies of $\alpha$; the inequality follows from the maximum principle. In view of the uniform convergences of $p_{-1 n}$, this implies that $\left|p_{-1}\right| \leqq \max _{\alpha}\left|p_{-1}\right|$ in $R-D$.

Similarly, $\left|p_{1 n}\right| \leqq \max _{\alpha}\left|p_{1 n}\right|$ in $R_{n}-D$. In fact, if $\left|p_{1 n}\right|=$ const. $=c_{n}$ on $\beta_{n}$ were $>\max _{\alpha}\left|p_{1 n}\right|$, then $\left|p_{1 n}\right|<c_{n}$ in $R_{n}-D$ and $\partial p_{1 n} / \partial n$ would not change its sign on $\beta_{n}$. Since $P_{1 n} \neq$ const., this derivative cannot vanish on the whole $\beta_{n}$, contrary to the condition $\int_{\beta_{n}} d p_{1 n}=0$. It follows that $\left|p_{1}\right| \leqq \max _{\alpha}\left|p_{1}\right|$ in $R-D$. Boundedness of $p_{-1}$ and $p_{1}$ on $R-D$ implies that of $p_{-1}-p_{1}$ on $R$.

Consider now the class of analytic functions $f+i \bar{f}$ on $R$ which have the development

$$
f+i \bar{f}=\sum_{1}^{\infty} b_{\nu} z^{\nu}, \quad \operatorname{Re}\left(b_{k}\right)=1,
$$

in $D$ with a single-valued real part on $R$. Set

$$
f_{0}+i \bar{f}_{0}=\frac{1}{\sigma_{P}}\left(P_{-1}-P_{1}\right) .
$$

TheOREM 3. The function $f_{0}$ gives to $D(f)$ the minimum

$$
\min D(f)=D\left(f_{0}\right)=2 \pi k / \sigma_{P} .
$$

Proof. Write

$$
f=\frac{p-p_{1}}{\sigma_{P}} \text { with } \alpha_{k}=\alpha_{-1 k} .
$$


Then, by (17), for $\lambda=1,-1$,

$$
D\left(p-p_{1}\right)-D\left(p-p_{-1}\right)=2 \pi k \sigma_{P}
$$

Therefore,

$$
D(f)=\frac{1}{\sigma_{P}^{2}} D\left(p-p_{1}\right)=\frac{2 \pi k}{\sigma_{P}}+D\left(f-f_{0}\right) .
$$

We add the following remark:

For all functions $p$ with $\int p d \bar{p}=0$ (for example $p_{1}$ and $p_{-1}$ ),

$$
D\left(p-p_{0}\right)=2^{-1} \pi k \sigma_{P}=\text { const. }
$$

This follows from (17) for $\lambda=0$.

THEOREM 4. For an arbitrary given Riemann surface $R$, the following properties are equivalent:

(a) The P-span vanishes.

(b) There are no functions $P$ with $\int p d p<0$ on $R$.

(c) All functions $P$ with $\int p d p \leqq 0$ are identical.

Proof. By (17),

$$
D\left(p-p_{0}\right)=\int p d \bar{p}+2^{-1} \pi k \sigma_{P} .
$$

Hence, (a) implies (b) and (c). Conversely, (a) follows from (b) or (c) by Theorem 2.

3. The class $\{Q\}$. So far we have dealt with single-valued functions $p$ without making any assumptions on the imaginary part. Consider now the subclass $\{Q\}$ of $\{P\}$ determined by the requirement that the period of the imaginary part $\bar{q}$ of $Q$ vanishes along every cycle dividing $R$ into two parts.

We note first the following simple statement:

Every open Riemann surface $R$ can be exhausted by compact sub-domains $R_{n}$, bounded by analytic Jordan curves, each of which divides $R$ into disjoint parts.

In fact, let first $\left\{R_{n}\right\}$ be any exhaustion on $R$, such that the complement $R-R_{n}$ consists of a finite number of noncompact domains $S_{n i}$. In $S_{n i}$, it is always possible to find a doubly connected domain $D_{n i}$ separating the common boundary part of $R_{n}$ and $S_{n i}$ from the common boundary part of $R$ and $S_{n i}$. Map. $D_{n i}$ conformally onto an annulus $\widetilde{D}_{n i}$. Take in $\widetilde{D}_{n i}$ a circle $\widetilde{\beta}_{n i}$. The corresponding curves $\beta_{n i}$ ( $n$ fixed) on $R$ are analytic Jordan curves bounding a subdomain $\widetilde{R}_{n} \supset R_{n}$ and each dividing $R$ into disjoint parts. By enlargement in the same way of any $R_{m}$ which contains $\tilde{R}_{n}$, we form $\widetilde{R}_{n+1}$. The sequence $\left\{\widetilde{R}_{n}\right\}$ is the desired exhaustion.

THEOREM 5. In $\{Q\}$, there is a unique principal function $Q_{\lambda}$ which mini- 
mizes $m_{\lambda}(q)$. Theorems 1-4 remain valid when $P$ and $P_{\lambda}$ are replaced by $Q$ and $Q_{\lambda}$. The coefficients $\alpha_{\lambda k}$ have to be taken with respect to $\{Q\}$ and the $P$-span replaced by the $Q-\operatorname{span} \sigma_{Q}=\alpha_{-1 k}-\alpha_{1 k}$.

Proof. If the boundary $\beta$ of $R$ consists of a finite number of analytic Jordan curves $\gamma_{i}$, replace $p_{1}$ in (7) by the function $q_{1}$ determined by

$$
q_{1}=\text { const. on } \gamma_{i} \text {. }
$$

Write $q_{-1} \equiv p_{-1}$. The above proofs will then be valid with $q$ and $q_{\lambda}$ substituted for $p$ and $p_{\lambda}$ respectively. If $R$ is arbitrary, let $\left\{R_{n}\right\}$ be an exhaustion, $R_{n}$ being bounded by a finite number of analytic Jordan curves each of which divides $R$ into disjoint parts. A function of the class $\{Q\}$ in $R$ or in $R_{n+1}$ then satisfies $\int d \bar{q}=0$ along each boundary curve of $R_{n}$ and the proof of Theorem 1 will hold verbatim.

In Theorems 2-4, replace $\sigma_{P}$ by $\sigma_{Q}$, and $f, f_{0}$ by $g, g_{0}$, respectively, the latter being subclasses of the former, satisfying $\int d \bar{g}=\int d \bar{g}_{0}=0$ along dividing cycles. Theorem 5 then follows.

Theorem 5 is illustrated by its application to planar surfaces (genus $=0$ ). The functions $Q$ are now single-valued analytic functions and the function $Q_{-1}-Q_{1}$ is bounded. Select $k=1$. Since, as is well known, $Q_{1}$ and $Q_{-1}$ map $R$ onto vertical and horizontal slit domains, $\sigma_{Q}$ is the span of $R$ (Schiffer [14]). Denote by $\bar{A}$ the complementary area in a schlicht mapping of $R$ by a function $Q$. ties:

THEOREM 6. For a planar surface $R$, we have the following extremal proper-

$1^{\circ}$. The vertical slit mapping $Q_{1}$ gives the minimum

$$
\min \left(2 \pi \alpha_{1}-\bar{A}\right)=2 \pi \alpha_{1,1} \text {. }
$$

The horizontal slit mapping $Q_{-1}$ gives the maximum

$$
\max \left(2 \pi \alpha_{1}+\bar{A}\right)=2 \pi \alpha_{-1,1} \text {. }
$$

$2^{\circ}$. The function $Q_{0}$ gives to $\bar{A}$ the maximum

$$
\max \int_{-\beta} q d \bar{q}=\int_{-\beta} q_{0} d \bar{q}_{0}=2^{-1} \pi \sigma_{Q} .
$$

$3^{\circ}$. The function $G_{0}=\left(1 / \sigma_{Q}\right)\left(Q_{-1}-Q_{1}\right)$ gives to the area $D(G)$ the minimum

$$
\min D(G)=D\left(G_{0}\right)=\frac{2 \pi k}{\sigma_{Q}}
$$

among all single-valued analytic functions

$$
G=\sum_{1}^{\infty} b_{\nu} z^{\nu}, \quad \operatorname{Re}\left(b_{1}\right)=1 .
$$


THEOREM 7. For planar Riemann surfaces, the following conditions are equivalent:

(a) The Q-span vanishes.

(b) $R$ is rigid, that is, schlicht mappings of $R$ are linearly dependent. In particular, $Q_{-1} \equiv Q_{1}$.

(c) $R$ can not be conformally mapped (by $Q$ ) onto a surface with $\int q d \bar{q}<0$.

(d) $\bar{A}=0$ in every schlicht mapping of $R$. $\{Q\}$.

Both theorems are immediate applications of Theorems 2-4 for functions

REMARK. For planar surfaces, the class $\{q\}$ for which $\int_{\beta} q d \bar{q} \leqq 0$ is a generalization of schlicht functions. We have, therefore, obtained for arbitrary Riemann surfaces results for functions which play for these surfaces the same role as the generalized schlicht functions play for planar surfaces. The author had stimulating discussions with Professor Schiffer on this subject.

4. Classification of Riemann surfaces. We conclude with an application to the classification of Riemann surfaces. The following abbreviations (cf. [9]) for certain properties of functions will be used:

$H$ harmonic single-valued nonconstant,

$K H$ with an imaginary part having no periods along dividing cycles,

$A$ analytic single-valued nonconstant,

$W$ meromorphic nonconstant,

$S$ schlicht single-valued nonconstant,

$B$ bounded,

$D$ with a finite Dirichlet integral,

$E$ omitting a set of values of positive area.

By a combination of letters, we denote classes of functions with the corresponding combination of properties. Let $O_{F}$ be the class of Riemann surfaces with an $F$-removable boundary (cf. [8]), that is, with no functions belonging to a given class $F$. We shall compare these classes mutually and with the classes $S_{P}$ and $S_{Q}$, defined by vanishing span:

$S_{P}$ the $P$-span vanishes,

$S_{Q}$ the $Q$-span vanishes.

THEOREM 8. For single-valued harmonic functions,

$$
O_{H B} \subset O_{H B D}=O_{H D}=S_{P} .
$$

Proof. The last equality is given by (22). Suppose now $S_{P}>0$. Then $p_{-1}$ $-p_{1} \neq$ const. and belongs to $H B D$.

The first two relations were found, by other methods, by Royden [7], the relation $O_{H B} \subset O_{H D}$ also by Virtanen [15]. The last relation is new, expressing the fact that there exist functions $H D$ if and only if the $P$-span is positive. 
It follows from Theorem 8 that the vanishing of the $P$-span is a conformally invariant property of the surface, i.e. independent of the location and order of the pole of the functions $\{P\}$.

THEOREM 9. For single-valued harmonic functions whose conjugates have no periods along dividing cycles,

$$
O_{K B} \subset O_{K B D}=O_{K D}=S_{Q} .
$$

Proof. The relations are obtained by a proof analogous to that of Theorem 8.

THEOREM 10. For planar surfaces,

$$
O_{A B} \subset O_{A B D}=O_{A D}=O_{S E}=S_{Q} .
$$

Proof. Since, for planar surfaces, $K=A$, the first two relations and the equality $O_{A D}=S_{Q}$ are included in Theorem 9 and written here for the sake of completeness. The last equality is given by (27) for $Q$ and by Schiffer's result on the univalency of $Q_{-1}+Q_{1}$.

The relations $O_{A B} \subset O_{A D}=O_{S E}$ were first proved by Ahlfors and Beurling [1] using the theory of extremal length. The last equality is due to Lehto [3].

We turn now back to arbitrary Riemann surfaces.

THEOREM 11. For analytic functions,

$$
O_{W E}=O_{A B} \subset O_{A D} .
$$

Proof. Suppose that a function of class $W E$ maps $R$ onto a surface $R^{\prime}$. Then, on the projection $\tilde{R}$ of $R^{\prime}$ on the complex $\tilde{z}$-plane, the function $\tilde{z} \in W E$. By Theorem 10, there is a function of class $A B$ on $\widetilde{R}$, hence on $R^{\prime}$ and finally on $R$. The opposite inequality is trivial and the first equality follows. An analogous reasoning shows that the existence of a function of class $A D$ on $R$ implies the existence of a function of class $A B$ on $R$.

Royden [7] was the first to remark on the generality of the inclusion $O_{A B} \subset O_{A D}$ for arbitrary surfaces, while Parreau [5] announced an independent proof. Later, also Lokki [4] proved it. The obvious extension of the relation $O_{W E}=O_{A B}$ to arbitrary surfaces seems to be new.

\section{LITERATURE}

1. L. Ahlfors and A. Beurling, Conformal invariants and function-theoretic null-seis, Acta Math. vol. 83 (1950) pp. 101-129.

2. H. Grunsky, Neue Abschätzungen zur konformen Abbildung ein- und mehrfach zusammenhängender Bereiche, Schr. Math. Semin. Univ. Berlin vol. 1 (1932) pp. 95-140.

3. O. Lehto, On the existence of analytic functions with a finite Dirichlet integral, Annales Academiae Scientiarum Fennicae Ser. A.I. no. 67 (1949) pp. 1-7.

4. O. Lokki, Beiträge zur Theorie der analytischen und harmonischen Functionen mit endlichem Dirichlet-integral, Annales Academiae Scientiarum Fennicae Ser. A.I. no. 92 (1951) pp. 1-10. 
5. M. Parreau, Sur certaines classes de fonctions analytiques uniformes sur les surfaces de Riemann, C. R. Acad. Sci. Paris vol. 231 (1950) pp. 751-753.

6. R. de Possel, Sur quelques propriétés de la représentation conforme des domains multiplement connexes, en relation avec le theorème des fentes parallèles, Math. Ann. vol. 107 (1932) pp. 496-504.

7. H. L. Royden, Some remarks on open Riemann surfaces, Annales Academiae Scientiarum Fennicae Ser. A.I. no. 85 (1951) pp. 1-8.

8. L. Sario, Ueber Riemannsche Flächen mit hebbarem Rand, Annales Academiae Scientiarum Fennicae Ser. A.I. no. 50 (1948) pp. 1-79.

9. - Sur la classification des surfaces de Riemann, Congr. Math. Scand. Trondheim, 1949, pp. 229-238.

10. - A linear operator method on arbitrary Riemann surfaces, Trans. Amer. Math. Soc. vol. 72 (1952) pp. 281-295.

11. - Construction of functions with prescribed properties on Riemann surfaces, Conference on Riemann Surfaces in Commemoration of the Hundredth Anniversary of Riemann's Inaugural-Dissertation, Princeton, 1951, Annals of Mathematics Studies (to appear).

12. - Minimizing operators on subregions, Proc. Amer. Math. Soc. (to appear).

13. - Capacity of the boundary and of a boundary component, Ann. of Math. (to appear).

14. M. Schiffer, The span of multiply connected domains, Duke Math. J. vol. 10 (1943) pp. 209-216.

15. K. I. Virtanen, Ueber die Existenz von beschränkten harmonischen Funktionen auf offenen Riemannschen Flächen, Annales Academiae Scientiarum Fennicae Ser. A.I. No. 75 (1950) pp. 1-8.

The Institute for Advanced Study, Princeton, N. J. 